\title{
Intravenous iron therapy for anemic cancer patients: a review of recently published clinical studies
}

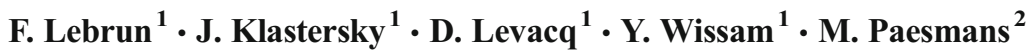

Received: 18 November 2016/Accepted: 14 March 2017 / Published online: 7 April 2017

(C) Springer-Verlag Berlin Heidelberg 2017

\begin{abstract}
Based on available literature and on the present review, IV iron administration to anemic cancer patients can increase significantly the level of $\mathrm{Hb}$, probably independently from the precise mechanism of anemia itself. However, in future studies, the benefit should be evaluated taking into account whether the anemia is due to absolute or functional iron deficiency; therapeutic modalities might be different for these two conditions. Along the same lines, it appears important to further evaluate the respective roles of PO and IV iron therapies and the modalities of their use in clinical practice. Until the results of such studies are available, it appears reasonable to propose IV iron therapy to anemic cancer patients as the resulting rise of $\mathrm{Hb}$ level may increase their quality of life and performance status and reduce the need for erythropoietinstimulating agents and/or blood transfusions.
\end{abstract}

Keywords Iron deficiency anemia $\cdot$ Cancer patients $\cdot$ Iron therapy $\cdot$ Erythropoiesis-stimulating agents

\section{Introduction}

Iron deficiency anemia has been recently thoroughly reviewed by Camaschella [1] and its two main clinical presentations:

J. Klastersky

jean.klastersky@bordet.be

1 Department of Medicine, Institut Jules Bordet, 1, Rue Héger-Bordet, 1000 Brussels, Belgium

2 Data Center, Institut Jules Bordet, 1, Rue Héger-Bordet, 1000 Brussels, Belgium iron deficiency anemia itself and iron-restricted erythropoiesis have been well defined from a physiological point of view. Actually, the presence of iron-deficiency (mainly due to blood losses) and that of anemia of chronic disorders (due to cancer, inflammation, and infection) are often associated in cancer patients. There are presently laboratory tests available for the evaluation of iron status and thus for, the identification of these two conditions and their possible combination (Tables 1 and 2). However, in cancer patients, anemia can be caused by several other conditions such as hemolysis, microangiopathy and chemotherapy-induced bone marrow failure; these etiologies for anemia in cancer patients are occasionally superimposed on iron-deficiency and/or chronic inflammation.

The prevalence of anemia in cancer patients is high. A paper by Maccis et al. [2] indicates that $63 \%$ of cancer patients were anemic; in that observational study, hemoglobin concentrations were inversely correlated with inflammatory markers, hepcidine, ferritin, erythropoietin, and reactive oxygen species. In another paper from Spain, by Steegman et al. [3], the prevalence of anemia was $48 \%$ and a high rate was associated with cisplatin-based chemotherapy, palliative chemotherapy, and with more than three lines of chemotherapy. Link and Schmitz [4] found a prevalence of hemoglobin level below $12 \mathrm{~g} / \mathrm{dl}$ of $49 \%$ and stressed that two thirds of these patients were not treated for anemia, which does not reflect current clinical practice guidelines, such as those recommended by ESMO (European Society of Medical Oncology) [5]. A similar conclusion was reached by de Castro et al. [6]. These authors looked for iron deficiency in patients with solid tumors; the overall prevalence of iron deficiency was 59\% (32\% were truly iron deficient and $27 \%$ were functionally deficient). Most non-anemic iron-deficient patients in that survey did not receive any form of iron supplementation before the start of chemotherapy. 
Table 1 Current laboratory tests for measurement of iron status (iron deficiency anemia IDA, anemia of chronic disease ACD)

\begin{tabular}{lccc}
\hline Test (unit) (Nl) & IDA & ACD & IDA + ACD \\
\hline Iron $(\mu \mathrm{Mol} / \mathrm{l})(10-30)$ & Low & Low & Low \\
Transferrin saturation $(\%)(>16$ to $<45)$ & $<16$ & Low-N & Low-N \\
Ferritin $(\mu \mathrm{g} / \mathrm{l})\left(40-300{ }^{-}\right)(20-200$ ) $)$ & $<10$ & $>100$ & $<100$ \\
Hemoglobin $(\mathrm{g} / \mathrm{dl})\left(>13 \delta^{-}\right)(>129)$ & Low & Low & Low \\
Mean corpuscular volume $(\mathrm{fl})(80-95)$ & $<80$ & Low-N & Low \\
Mean corpuscular hemoglobin $(\mathrm{pg})(27-34)$ & $<27$ & Very low & Low \\
\hline
\end{tabular}

In a seminal paper by Aapro et al. [7], the prevalence of iron deficiency in different cancer populations was found 29$60 \%$. That paper also stressed that IV iron supplementation of erythropoietic stimulating agents reduced the mean dose required with, in addition, adequate cost-effectiveness and enhanced response rates to these agents. It also presented some evidence on the use of IV iron therapy as a first-line therapy and suggested that some patients could benefit from IV iron even without concomitant erythropoietic stimulating agents. Along the same lines, Steinmetz et al. [8] published their experience with ferric carboxymaltose in the treatment of cancer and chemotherapy-associated anemia, in 639 patients. Patients with baseline hemoglobin up to $11 \mathrm{~g} / \mathrm{dl}$ and serum ferritin up to $500 \mathrm{ng} / \mathrm{ml}$ benefited from the treatment as well as patients with ferritin $>500 \mathrm{ng} / \mathrm{ml}$ and low transferrin saturation. The substantial $\mathrm{Hb}$ increase and stabilization of hemoglobin at 11-12 $\mathrm{ng} / \mathrm{dl}$ suggests a role for IV iron alone for the correction of anemia in those patients. At the same time, Petrelli et al. [9] performed a meta-analysis of controlled trials comparing parenteral or oral iron versus no iron, when added to erythropoiesis-stimulating agents in patients with chemotherapy-induced anemia. They showed that parenteral iron reduced the risk of transfusions by $23 \%$ and increased the chance of hematopoietic response by $29 \%$ when compared with erythropoiesis-stimulating agents alone. On the contrary, oral iron did neither increase the hematopoietic response nor the transfusion rate. Similar conclusions were reached by Gavter-Gvili et al. [10] who reported a systematic review and meta-analysis of randomized controlled trials of IV iron supplementation for the treatment of chemotherapy-induced anemia; they concluded that IV iron added to erythropoiesisstimulating agents resulted in an increase of hematopoietic response and reduction of the need of transfusions, with no difference in mortality of adverse events. The mechanism by which iron enhances the action of erythropoiesis-stimulating agents has not been completely elucidated. Increased iron in macrophages might lead to overexpression of ferraportin which in turn would increase the mobilization of iron for erythropoiesis.

The pros and cons for oral iron therapy versus IV iron therapy have been nicely discussed by Camaschella [1], although it was not focused on cancer patients, in whom the situation is usually more complex than in non-cancer patients, as already mentioned. Actually, we lack a predictive test to identify patients who are expected to respond to oral iron therapy; the oral iron challenge test is rarely used, although a recent study by Kobume et al. [11] suggests its potential value; on the other hand, the measurement of serum hepcidin is still not yet standardized enough to be used in routine practice, although there are indications that high hepcidin levels predict for functional iron-deficiency as shown by Durigova et al. [12] for which oral iron is probably not efficacious as discussed by Steinmetz [13] and who also concluded "that there is still an urgent need for trials investigating diagnostic approaches for the optimal tailoring of iron... in cancer patients with anemia."

\section{Review of recently published studies}

In that context, we decided to review the published papers about the use of iron therapy for anemia in cancer patients. We made a systematic research and thus retrieved all the available papers, using the following keywords: iron therapy and
Table 2 Proposed laboratory tests for measurement of iron status (IDA iron deficiency anemia, ACD anemia of chronic disease)

\begin{tabular}{|c|c|c|c|}
\hline Test (unit) (Nl) & IDA & $\mathrm{ACD}$ & $\mathrm{IDA}+\mathrm{ACD}$ \\
\hline Soluble transferrin receptors $(\mathrm{sTFR})(\mathrm{mg} / \mathrm{l})^{\mathrm{a}}$ & High & Low-N & Variable \\
\hline sTFR/log ferritin index ${ }^{a}$ & $>2$ & $<1$ & $>2$ \\
\hline Hepcidin $^{\mathrm{a}}$ & Very low & High & N-high \\
\hline Reticulocyte $\mathrm{Hb}$ content $(\mathrm{pg})(31.2 \pm 1.6)$ & Low & Low & Low \\
\hline Perls staining for bone marrow iron (positive) & Negative & Strongly positive & Positive \\
\hline
\end{tabular}

${ }^{\text {a }}$ Vary according to method 
anemia and cancer, on PubMed NCBI starting in 2013 up to now. We chose to start our search in 2013 because Steinmetz et al. [8] published in 2013 the largest study so far on the role of IV iron for the treatment of anemia in cancer, and suggested that IV iron alone, without the erythropoiesis-stimulating agents might have a significant role. He also analyzed two other studies using IV iron for anemic cancer patients, both showing a clear benefit in terms of transfusion reduction of that approach, in comparison with either no iron substitution or oral iron [14, 15]. Actually, in 2012, Steinmetz had reviewed the topic of IV iron in the treatment of anemia in cancer patients [13] on the basis of published studies. We thus reviewed all other studies that have been published since 2013 on that topic. We found seven studies in which IV iron was used in anemic cancer patients. Table 3 summarizes our findings regarding the type of each study, inclusion and evaluation criteria as well as the conclusions proposed by the authors.

Abdel-Razeq [16] investigated IV iron (ferric sucrose) monotherapy for the treatment of non-iron-deficiency anemia in cancer patients undergoing chemotherapy and/or radiotherapy. In that pilot study, in 23 patients, with a mean baseline hemoglobin level of $9.6 \mathrm{~g} / \mathrm{dl}$, the mean change in hemoglobin level was $1.7 \mathrm{~g} / \mathrm{dl}$ for those patients who completed at least nine treatments (200 mg weekly) and $2.1 \mathrm{~g} / \mathrm{dl}$ in those who completed the full 12-week treatment, a highly statistically significant difference $(p=0.0007)$. Five patients were transfused and considered as failures. No treatment-related adverse effects were noted.

Keeler et al. [17] evaluated the feasibility and clinical efficacy of IV iron, for preoperative anemia in patients with colorectal cancer (a single $1000 \mathrm{mg}$ of ferric carboxymaltose). There was a significant $(p<0.001)$ median increase in hemoglobin levels of $1.8 \mathrm{~g} / \mathrm{dl}$. Five patients received transfusions until the 4th postoperative day, which was fewer than predicted.

Hedenus et al. [18] published a small randomized study of IV iron (ferric carboxymaltose, $1000 \mathrm{mg}$ ) versus control (no iron) in 17 patients with functional iron deficiency and lymphoid malignancies undergoing chemotherapy. All treated patients achieved a hemoglobin increase $>1 \mathrm{~g} / \mathrm{dl}$ significantly higher than that of controls $(p=0.02)$. No treatment-related events were reported.

Calleja et al. [19] conducted a multicenter, observational study of ferric carboxymaltose in patients with colon cancer and anemia; 111 patients received IV iron and 155 were no IV iron subjects. There was a significant reduction for the need of transfusion in the cohort receiving IV iron (9.9 vs $38.7 \%$, $p<0.001)$. Responses in terms of hemoglobin levels were significantly higher among the treated patients who also had a lower number of reinterventions, post-surgery complications, and a shorter length of stay in hospital.

Toledano et al. [20] evaluated the clinical use of carboxymaltose (median dose per patient $1000 \mathrm{mg}$ ) in 367 patients with solid tumors or hematological malignancies. The median hemoglobin level improved from 10.3 to $11.8 \mathrm{~g} /$ $\mathrm{dl}$; that increase was the same in patients treated with iron alone or with additional erythropoiesis-stimulating agents (34\% of patients). No adverse reactions and no hypersensitivity reactions were observed.

In addition to these studies dealing with cancer patients and anemia, caused by digestive cancers prior to an operation, or chemotherapy in metastatic solid tumors or hematological malignancies, we found three studies on the use of IV iron supplementation in patients after surgery for gastric, colon, and other cancers.

Yoon et al. [21] compared the administration of IV iron to 63 patients who became anemic (hemoglobin $<9 \mathrm{~g} / \mathrm{dl}$ ) after gastrectomy for cancer and to outcome in 60 patients without treatment (observation group). The slope of the changes in the hemoglobin level was significantly higher in the iron group than in the observation one $(0.65$ vs $0.35, p<0.001)$. The postoperative hospital stay was significantly longer in the iron group but no differences in surgical complications were seen.

Titos-Arcos et al. [22] evaluated IV iron in 71 paired patients after colon cancer surgery. The mean hemoglobin levels were similar in both groups and the rate of blood transfusions was also similar (30\%).

Ferrari et al. [23] reported on the use of oral ferrous bisglycinate or oral ferrous sulfate in patients with various operated solid tumors and mild anemia (hemoglobin value between 10 and $12 \mathrm{~g} / \mathrm{dl}$ ) The increase in the hemoglobin level were $1.4 \mathrm{~g} / \mathrm{dl}$ in both groups, after 2 months of therapy. Adverse events (GI upsetting) were respectively seen in two and four patients; all were grade 1.

The laboratory data relative to the inclusion and the evaluation of the patients included in all these studies are summarized in Table 3, as well as other demographical and technical data. For each study, we proposed conclusions and comments that will be further addressed in the discussion.

\section{Safety of IV iron}

Most studies discussed in this review were performed with carboxymaltose. An extensive review of ferric carboxymaltose by Keating [24] is available. It indicates that it is well tolerated, with a low risk of hypersensitivity reactions; it was overall better tolerated than oral ferrous sulfate, mainly because of a lower incidence of gastrointestinal side effects. The most common laboratory adverse effect was transient and asymptomatic hypophosphatemia. As that review mainly deals with non-cancer patients, these conclusions might not fully apply to our population. In our review of the seven recent studies involving IV administered iron, two studies did not mention adverse effects; in the five other studies, no cases of adverse effects linked to the administration was 


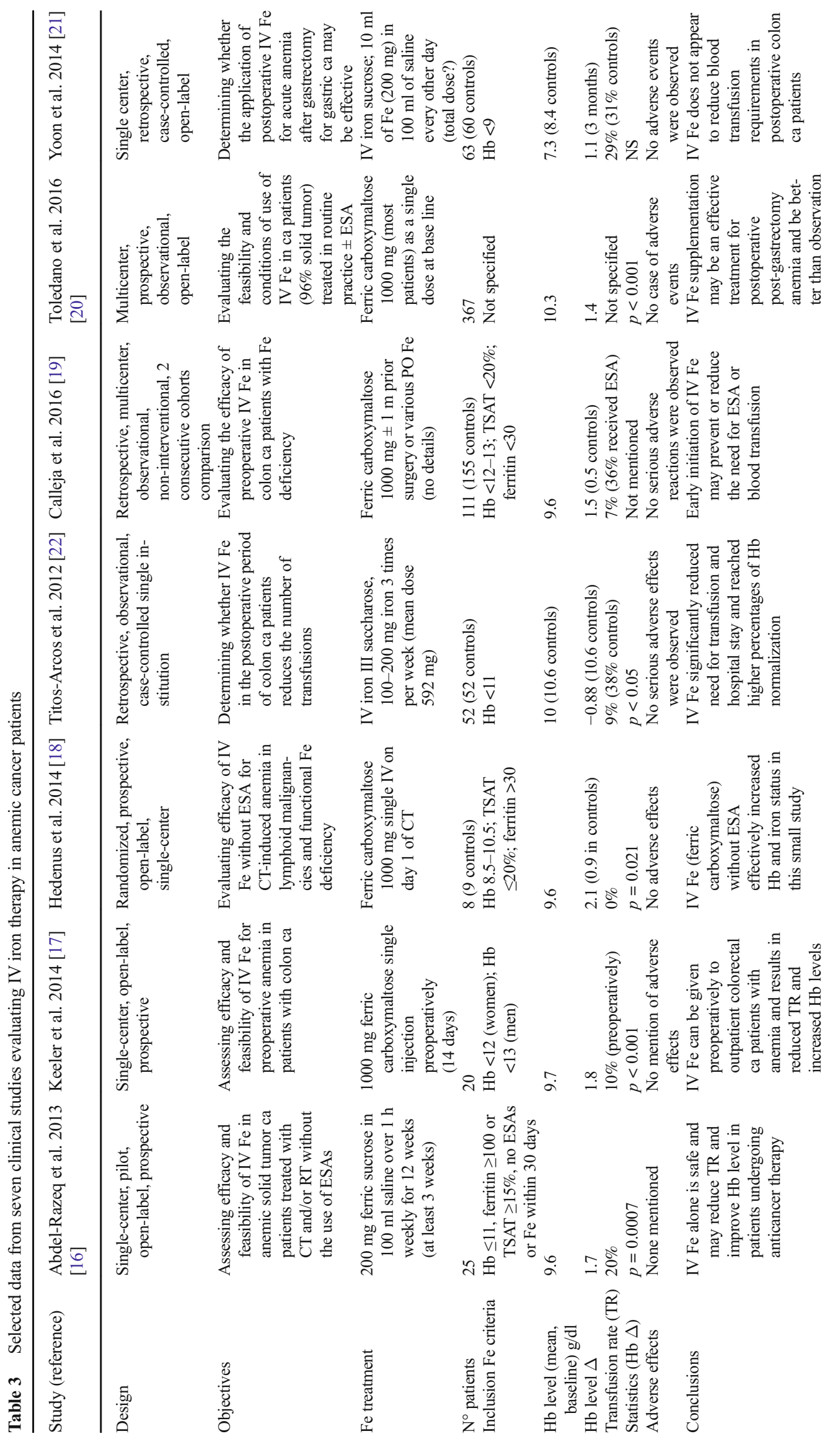


reported; in particular, no allergic or anaphylactic reactions did occur (see Table 3). In their masterful review on the prevalence and management of cancer-related anemia, iron deficiency and the specific role of iron, Aapro et al. [7] discussed in details the tolerability of IV iron in clinical routine and also concluded to a low incidence of hypersensitivity reactions, when the dextran preparations are avoided. They also discussed the potential effect of iron in increasing the risk of infection; this is obviously an important issue in cancer patients but no evidence for it has been documented so far. Equally important is their discussion of a possible interaction between iron and some chemotherapies and the theoretical possibility that iron might induce cancer or increase the risk of serious infection in cancer patients.

As far as infections are concerned, the role of increased iron availability probably benefits to certain bacterial and fungal pathogens, namely mucormycosis [25]; iron homeostasis and macrophage function appear to be interrelated in an adaptive advantage to combat invading microbes by decreasing iron availability. Although there are many experimental data to support such a hypothesis, these experimental models do not translate easily into human clinical conditions and should be applied with caution [26, 27]. As far as a possible effect of high iron availability on cancer growth is concerned, the most often cited situation is that of hepatocellular carcinoma in chronic liver disease due to hemochromatosis and/or thalassemia. An independent association between serum ferritin and hepatocellular carcinoma has been also recently shown in chronic liver disease of viral etiology [28]. A comprehensive review about the safety of iron dextran infusion in cancer patients [29] concluded that no clinical data support an increased risk of iron overload on the growth of cancer, although such a possibility cannot be excluded. Ultimately, clinicians should balance the immediate benefits of iron administration against its possible long-term unknown risks.

\section{Discussion}

As already stated, the overall prevalence of iron deficiency anemia is high in cancer patients: $59 \%$ (32\% with absolute iron deficiency and $27 \%$ with functional iron deficiency) and has a significant negative impact on the already impaired quality of life of these patients $[30,31]$. Nonetheless, the problem is underestimated as iron parameters are not commonly measured in newly diagnosed cancer patients, although possible therapies are available such as erythropoietin-stimulating agents and iron therapy, namely IV administration of iron [32]. Because caveats have been expressed regarding the use of erythropoietin-stimulating agents, we reviewed recent published data about the use of IV iron for suspected iron deficiency anemia in cancer patients.
We found seven studies of the use of IV iron in anemic cancer patients since the study by Steinmetz et al. [8] which suggested that such an approach might be effective, even without erythropoietin-stimulating agents. A summary of these seven studies is presented in Table 3.

The designs of these studies are highly variable, making a global analysis impossible and representing a major limitation of this review. Another limitation is that quality of life was not assessed in those studies neither we could find a systematic evaluation of clinically significant outcomes. Only in one study [19] it was mentioned that IV iron therapy significantly reduced the duration of the hospital stay. Thus, we had to rely on laboratory surrogates that were available in all seven studies. In all these studies, patients were anemic before treatment (mean $\mathrm{Hb}$ level at baseline $9.5 \mathrm{~g} \%$ ) and in all the level of $\mathrm{Hb}$ was reevaluated after therapy (in most after a month). With only one exception [22], a statistically significant increase of the $\mathrm{Hb}$ level was observed (mean $\mathrm{Hb}$ increase: $1.6 \mathrm{~g} \%$ ). That increase of the $\mathrm{Hb}$ level was clearly superior to that observed in controls in two studies [18, 19]. Improvement in other iron metabolism parameters were reported as well, but the available information is insufficient for a global analysis. The effect of the rise of the $\mathrm{Hb}$ level on the quality of life of the patients was rarely reported and could not be evaluated.

Another parameter for the efficacy of iron therapy might be a reduction of the need for blood transfusion but it requires control data. In our review, we had only two such studies both in operated colon cancer patients. In anemic patients receiving IV iron preoperatively, there was a significant reduction in the need of transfusion [19]; in a study where patients received IV iron postoperatively, no benefit was observed [22].

In most of the studies reported here, no sufficient data are provided to separate between absolute and functional iron deficiency and it is likely that the iron deficiency anemia was multifactorial in many patients, namely blood losses in the surgical series $[17,19,21,22]$. Therefore, it is not surprising that iron supplementation was effective, although the only negative study [22] was conducted in operated colon cancer patients. Two of these studies addressed specifically anemic cancer patients whose anemia was likely due to functional iron deficiency [16, 18]; although both are small studies, a significantly positive result in terms of $\mathrm{Hb}$ rise was reported. Future studies should clearly evaluate the respective contribution of absolute and functional iron deficiency, perhaps using systematically the evaluation of hepcidin serum levels [33].

In four studies reviewed here [17-20], ferric carboxymaltose has been used while in the three others, ferric sucrose was the formulation. It is important to stress that no adverse effects were reported with either iron preparation, which confirms data available from the literature [24].

The overall conclusions made from the review of these studies are summarized in Table 3; in six out of seven, it was concluded that IV iron administration increased the $\mathrm{Hb}$ 
level in anemic cancer patients. The question whether PO iron therapy would have been similarly effective remains speculative. A thorough discussion of that question is available in Camaschella's review [1] but does not address specifically cancer patients. The question of PO iron therapy in anemic cancer patients has also been discussed by Aapro et al. [7] and Steinmetz [13]; in both reviews, the authors doubt about the efficacy of PO iron for functional iron deficiency and acknowledge the higher rate of side effects with oral preparations. We found one relatively recent paper [23] comparing the efficacy of two PO iron preparations in iron deficiency anemia in cancer patients; both were effective but associated with a substantial (17-33\%) rate of side effects. As the selected patients had to have ferritin levels $<30 \mathrm{ng} / \mathrm{ml}$, it can be suspected that most of the patients had not a functional iron deficiency. Therefore, in future studies, it might be useful to test for PO iron efficacy, using standardized and recognized techniques [34], before turning to IV iron administration which is technically more demanding and, in any case, more expensive.

To conclude with, based on available literature and on the present review, IV iron administration to anemic cancer patients can increase significantly the level of $\mathrm{Hb}$, probably independently from the precise mechanism of anemia itself. However, in future studies, the benefit should be evaluated taking into account whether the anemia is due to absolute or functional iron deficiency; therapeutic modalities might be different for these two conditions. Along the same lines, it appears important to further evaluate the respective roles of PO and IV iron therapies and the modalities of their use in clinical practice. Until the results of such studies are available, it appears reasonable to propose IV iron therapy to anemic cancer patients as the resulting rise of $\mathrm{Hb}$ level may increase their quality of life and performance status and reduce the need for erythropoietinstimulating agents and/or blood transfusions.

\section{Compliance with ethical standards}

Conflict of interest None of the authors declares a conflict of interest. This study was supported by a scientific grant from VIFOR SA to the Institut Jules Bordet. The authors declare to have the full control of all primary data. They agree to allow the journal to review their data if requested.

\section{References}

1. Camaschella C (2015) Iron-deficiency anemia. N Engl J Med 372: 1832-1843

2. Macciò A, Madeddu C, Gramignano G et al (2015) The role of inflammation, iron, and nutritional status in cancer-related anemia: results of a large, prospective, observational study. Haematologica 100:124-132

3. Steegman JL, Sánchez Torres JM, Colomer R et al (2013) Prevalence and management of anaemia in patients with non- myeloid cancer undergoing systemic therapy: a Spanish survey. Clin Transl Oncol 15:477-483

4. Link H, Schmitz S (2013) Treatment of cancer-associated anaemia: results from a two-day cross-sectional survey in Germany. Onkologie 36:266-272

5. Schrijvers D, De Samblanx H, Roila F, on behalf of the ESMO Guidelines Working Group (2010) Erythropoiesis-stimulating agents in the treatment of anaemia in cancer patients: ESMO clinical practice guidelines for use. Ann Oncol 21:v244-v247

6. de Castro J, Gascón P, Casa A, Muñoz-Langa J et al (2014) Iron deficiency in patients with solid tumours: prevalence and management in clinical practice. Clin Transl Oncol 9:823-828

7. Aapro M, Österborg A, Gascón P et al (2012) Prevalence and management of cancer-related anaemia, iron deficiency and the specific role of i.v. iron. Ann Oncol 23:1954-1962

8. Steinmetz T, Tschechne B, Harlin O et al (2013) Clinical experience with ferric carboxymaltose in the treatment of cancer- and chemotherapy-associated anaemia. Ann Oncol 24:475-482

9. Petrelli F, Borgonovo K, Cabiddu M et al (2012) Addition of iron to erythropoiesis-stimulating agents in cancer patients: a meta-analysis of randomized trials. J Cancer Res Clin Oncol 138:179-187

10. Gafter-Gvili A, Rozen-Zvi B, Vidal L et al (2013) Intravenous iron supplementation for the treatment of chemotherapy-induced anaemia-systematic review and meta-analysis of randomized controlled trials. Acta Oncol 52:18-29

11. Kobune M, Miyanishi K, Takada K et al (2011) Establishment of a simple test for iron absorption from the gastrointestinal tract. Int $\mathrm{J}$ Hematol 93:715-719

12. Durigova A, Lamy PJ, Thezenas S et al (2013) Anemia and iron biomarkers in patients with early breast cancer. Diagnostic value of hepcidin and soluble transferrin receptor quantification. Clin Chem Lab Med 51:1833-1841

13. Steinmetz HT. The role of intravenous iron in the treatment of anemia in cancer patients. Ther Adv Hematol 2012; 0:1-15

14. Dangsuwan P, Manchana T (2010) Blood transfusion reduction with intravenous iron in gynecologic cancer patients receiving chemotherapy. Gynecol Oncol 116:522-525

15. Kim YT, Kim SW, Yoon BS et al (2007) Effect of intravenously administered iron sucrose on the prevention of anemia in the cervical cancer patients treated with concurrent chemoradiotherapy. Gynecol Oncol 105:199-204

16. Abdel-Razeq H, Abbasi S, Saadi I et al (2013) Intravenous iron monotherapy for the treatment of non-iron-deficiency anemia in cancer patients undergoing chemotherapy: a pilot study. Drug Design, Development and Therapy 7:939-944

17. Keeler BD, Simpson JA, Ng S et al (2014) The feasibility and clinical efficacy of intravenous iron administration for preoperative anaemia in patients with colorectal cancer. Color Dis 16:794-800

18. Hedenus M, Karlsson T, Ludwig H et al (2014) Intravenous iron alone resolves anemia in patients with functional iron deficiency and lymphoid malignancies undergoing chemotherapy. Med Oncol 31:302

19. Calleja JL, Delgado S, del Val A et al (2016) Ferric carboxymaltose reduces transfusions and hospital stay in patients with colon cancer and anemia. Int J Color Dis 31:543-551

20. Toledano A, Luporsi E, Morere JF et al (2016) Clinical use of ferric carboxymaltose in patients with solid tumours or haematological malignancies in France. Support Care Cancer 24:67-75

21. Yoon HM, Kim YW, Ham BH et al (2014) Intravenous iron supplementation may be superior to observation in acute isovolemic anemia after gastrectomy for cancer. World J Gastroenterol 20: $1852-1857$

22. Titos-Arcos JC, Soria-Aledo V, Carrillo-Alcaraz A et al (2012) Is intravenous iron useful for reducing transfusions in surgically treated colorectal cancer patients? World J Surg 36:1893-1897

23. Ferrari P, Nicolini A, Manca ML et al (2012) Treatment of mild non-chemotherapy-induced iron deficiency anemia in cancer 
patients: comparison between oral ferrous bisglycinate chelate and ferrous sulfate. Biomed Pharmacother 66:414-418

24. Keating GM (2014) Ferric carboxymaltose: a review of its use in iron deficiency. Drugs. doi:10.1007/s40265-014-0332-3

25. Ibrahim AS, Spellberg B, Walsh TJ et al (2012) Pathogenesis of mucormycosis. CID 54:S16-S22

26. Nairz M, Schroll A, Demetz E et al (2015) 'Ride on the ferrous wheel' - the cycle of iron in macrophages in health and disease. Immunobiology 220:280-294

27. Dadwal SS, Tegtmeier B, Liu X et al (2014) Impact of pretransplant serum ferritin level on risk of invasive mold infection after allogeneic hematopoietic stem cell transplantation. Eur J Heamatol 94: 235-242

28. Patil PS, Mohandas KM, Bhatia SJ et al (2014) Serum ferritin and the risk of hepatocellular carcinoma in chronic liver disease of viral etiology: a case-control study. Indian J Gastroenterol 33:12-18
29. Gilreath JA, Stenehjem DD, Rodgers GM (2012) Total dose iron dextran infusion in cancer patients: is it safe? JNCCN 10:669-676

30. De Castro J, Gascòn P, Casas A et al (2014) Iron deficiency in patients with solid tumours: prevalence and management in clinical practice. Clin Transl Oncol 16:823-828

31. Cella D, Kallich J, McDermott A et al (2004) The longitudinal relationship of hemoglobin, fatigue and quality of life in anemic cancer patients: results from five randomized clinical trials. Ann Oncol 15:979-986

32. Laï-Tiong F, Brami C, Dubroeucq O et al (2016) Management of anemia and iron deficiency in a cancer center in France. Support Care Cancer 24:1091-1096

33. Fraenkel PG. Understanding anemia of chronic disease. American Society of Hematology

34. Van Santen S, de Mast Q, Oosting ID et al (2014) Hematologic parameters predicting a response to oral iron therapy in chronic inflammation. Haematologica 99:e171-e173 